\title{
Pompage d'Ekman dans un écoulement de marée : génération d'un courant résiduel
}

\author{
D. Aelbrecht \& G. Chabert d'Hières \\ Laboratoire des Ecoulements Géophysiques et Industriels \\ Institut de Mécanique de Grenoble, C.N.R.S., URA 1509
}

\section{Introduction}

L'action des courants sur le transport solide est diverse. Dans une mer macrotidale comme la Manche, les forts courants associés à la marée, apparemment alternatifs au premier ordre, peuvent engendrer des circulations résiduelles du second ordre. Cette notion de circulation résiduelle est particulièrement cruciale pour la maitrise des transports sédimentaires (ou de pollution), puisqu'elle en détermine les évolutions à long terme. Le vent est lui aussi un vecteur potentiel de courant permanent. Nous insisterons sur le caractère lagrangien (différent de l'approche eulérienne) lié au suivi des masses d'eau marquées par des sédiments ou des polluants (notion de dérive de Stokes).

Les modes de génération des courants résiduels par la marée sont multiples. Parmi cet ensemble, on peut distinguer plusieurs processus :

- la dissymétrie " habituelle » des courants de flot et de jusant due à la non-linéarité de l'onde de marée ;

- l'effet dissymétrique du frottement suivant que l'on se trouve à haute ou basse mer. En général, le flot accompagne le montant. Le jusant accompagne le baissant, et par conséquent « frotte » plus :

\begin{abstract}
- les variations topographiques (bancs de sable, rupture du plateau continental, estran) sont générateurs d’un comportement fortement non-linéaire de l'écoulement tendant à une "rectification " de celui-ci (ZIMMERMAN, 1978: GarreaU \& MAZE. 1992);

- les courants associés à la pente longitudinale moyenne (sur une période de marée) de la surface libre dans le sens de propagation de l'onde de marée (écoulement gravitaire moyen) :

- les effets de caps peuvent engendrer la formation de tourbillons locaux résiduels :

- les courants permanents de densité associés à la possible formation de front côtier due aux apports fluviatiles ;

- et l'effet de la rotation terrestre associée au frottement de fond en présence d'une limite latérale (côte) dans un écoulement de marée parallèle à cette limite sont des conditions favorables au développement d'un pompage d'Ekman. Dans l'hémisphère Nord, le courant de fond est dévié vers la gauche de l'écoulement principal par effet du frottement sur l'équilibre entre le gradient de pression et la force de Coriolis (couche d'Ekman). La limite latérale impose des mouvements verticaux, et par continuité, une composante horizontale perpendiculaire à la
\end{abstract}

\section{Ekman pumping in a tidal motion : generation of a rectified current}

Among the potential sources of suspended materials transport, tidal currents obviously play an important role : firstly due to the usual asymetry between flux and ebb (non-linearity of the tidal wave), but also because of the coastal rectification of a purely sinusoidal offshore current, which tends to the generation of a residual circulation. This process comes out from the modification of the lateral boundary layer by the "Ekman pumping ". We make a physical modelling of this motion on the large rotating tank of the Institut de Mécanique de Grenoble and study the hydrodynamic behaviour by varying the main parameters of the flow. The results of this work may be applied to the french coasts of Pas-de-Calais, near Boulogne s/mer. 
côte, modifiant la structure du champ de quantité de mouvement horizontale suivant que l'écoulement parallèle laisse la côte à sa droite ou à sa gauche (AELBRECHT et al., 1993). C'est précisément ce dernier processus que nous nous limiterons ici à étudier.

L'objectif général de cette étude est donc de décrire (mesurer, quantifier, expliquer) la structure du courant résiduel, résultat d'un écoulement parallèle à un mur droit vertical, sur un fond plat incliné ou non, dans un fluide homogène. Pour cela, nous utilisons la grande plaque tournante de l'Institut de Mécanique de Grenoble dans laquelle nous faisons osciller un fond mobile (fig. 2). Ce dispositif ne nous sert pas à reproduire la marée et tous ses effets, mais de mettre en évidence expérimentalement le processus particulier lié au pompage d'Ekman. Les côtes du Pas-de-Calais, de l'embouchure de la Somme au cap Gris-Nez (voir fig. I), correspondent bien à la configuration que nous avons décrite pour notre modèle physique, même si on y retrouve toutes les autres sources possibles de circulations résiduelles énoncées ci-avant : variations topographiques (bancs de sable), apports fluviatiles, effet du vent...

SAlomon et Breton (1991) ont calculé un modèle hydrodynamique bidimensionnel de la Manche montrant la circulation résiduelle générale sous différentes conditions de vent. Mais pour décrire convenablement le processus que nous étudions, il nous faut apprécier la structure tridimensionnelle des courants de marée. PRANDLE (1982) a étudié la structure verticale de ces courants dans le cas d'un fond plat horizontal infini (pas de limites latérales) et a proposé quelques solutions analytiques pour différentes formes de viscosité verticale turbulente (constante ou linéairement dépendant avec la profondeur). MAAS et VAN HAREN (1987) ont repris cette analyse appliquée aux courants de marée et d'inertie et l'ont comparée à leurs observations en mer du Nord, mais sans approcher la question des courants résiduels. TEE (1979 et 1980) a calculé les courants associés à une marée se propageant perpendiculairement à la côte, en terme de courants oscillants (du premier ordre) d'une part, et de courants résiduels (du second ordre) d'autre part. Il calcule d'abord un champ de vitesse intégrée sur la colonne d'eau, puis, doté d'un modèle de distribution verticale, il détermine la structure 3-D de ces courants. Mais il n'introduit pas dans la zone côtière de son modèle les termes de frottement latéral, qui interviennent dans le processus que nous décrivons.

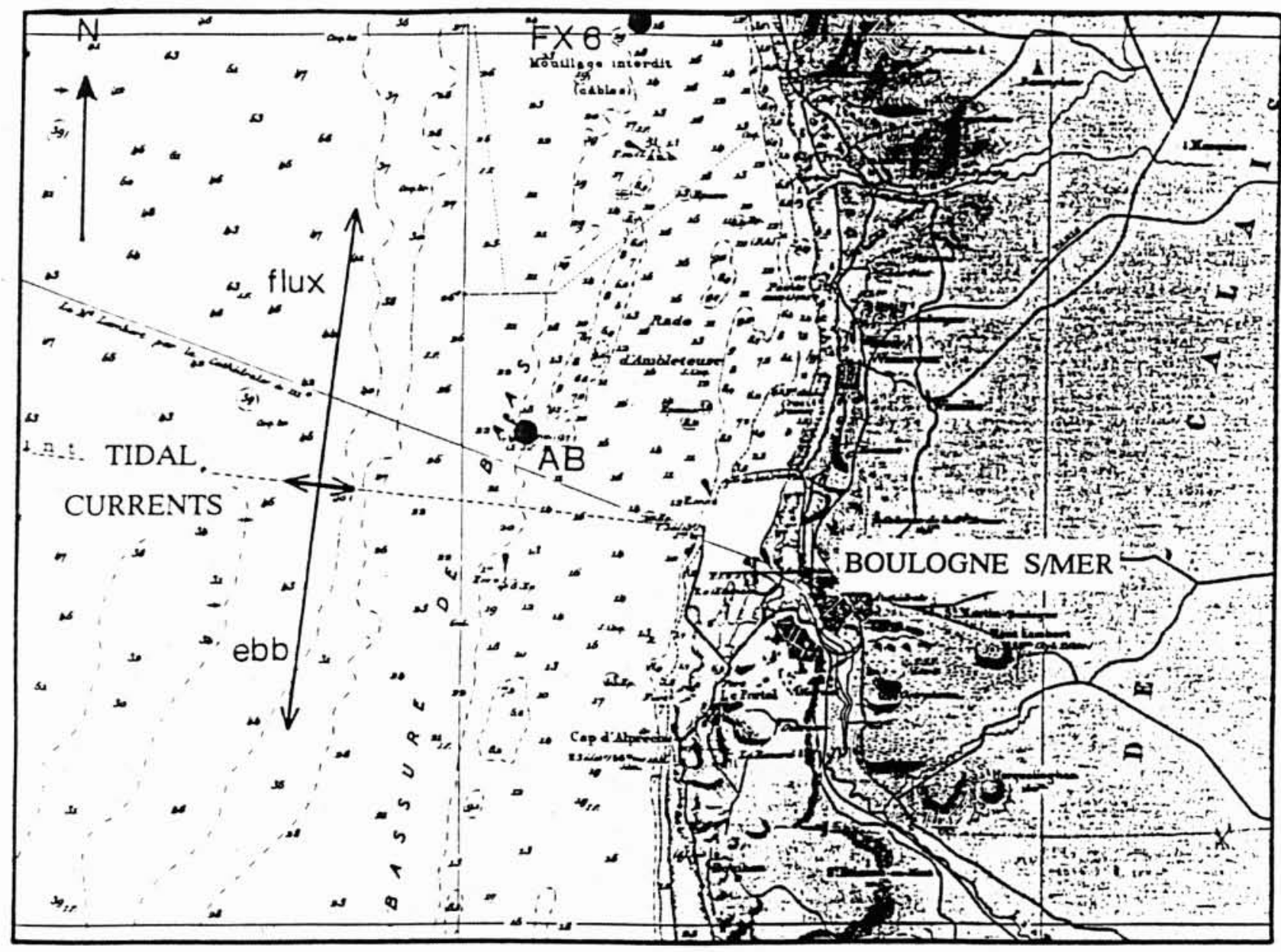

1. Carte bathymétrique aux environs de Boulogne s/mer (tirée de la carte SHOM 5107). 


\section{Expérience}

\subsection{Dispositif expérimental. Mesures (voir fig. 2)}

Nous nous plaçons dans un système de coordonnées $(O x y z)$, tournant autour de l'axe $(O z)$ avec une vitesse angulaire $f / 2$ ( $f$ est le paramètre de Coriolis), l'axe $(O x)$ est parallèle au mur vertical, $(O y)$ étant orthogonal à celui-ci. Le mouvement relatif de l'eau est obtenu par un mouvement d'oscillation du fond mobile. Ce fond mobile, d'une forme rectangulaire de $8 \mathrm{~m}$ sur $2 \mathrm{~m}$, se déplace sur un champ de roulements à la vitesse $U(t)=U_{0} \cos (\sigma t)$ à l'aide d'un moteur piloté par ordinateur et muni d'une régulation de position. Les paramètres dimensionnels de l'écoulement sont: la profondeur $H(y)$, constante ou variant linéairement avec $y$ (fond horizontal ou incliné); l'angle d'inclinaison du fond mobile, $\alpha$; le paramètre de Coriolis, $f=2 \Omega$ (la période de rotation est alors $T=2 \pi / \Omega$ ) ; la fréquence d'oscillation du fond mobile $\sigma$ (période d'oscillation $\tau=2 \pi / \sigma$ ) ; et l'amplitude du courant oscillant $U_{0}$. Nous supposons l'écoulement uniforme selon I'axe $(O x)$, hypothèse convenable étant donnée la dimension du fond par rapport à l'élongation maximale de celui-ci pendant une oscillation. Dans ces conditions, l'écoulement est contrôlé par les nombres adimensionnels suivants :

$R_{a t}=\sigma / f=$ Inertie temporelle/Coriolis nombre de Rossby temporel

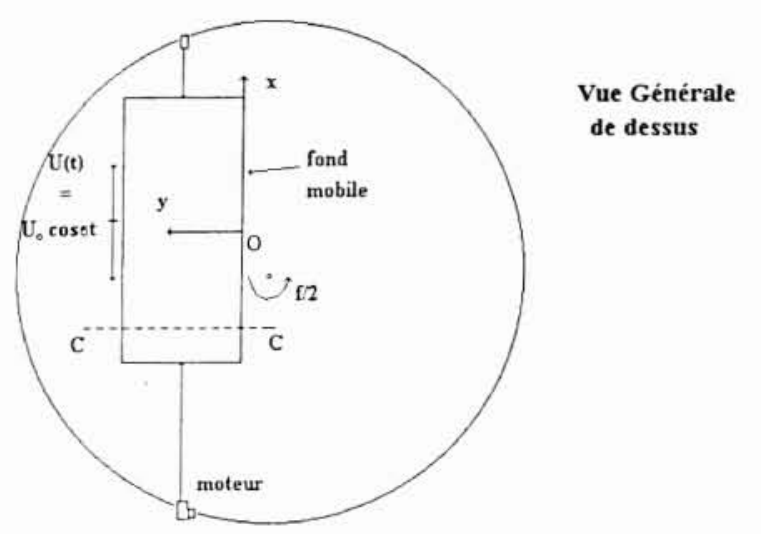

coupe $\mathrm{CC}$

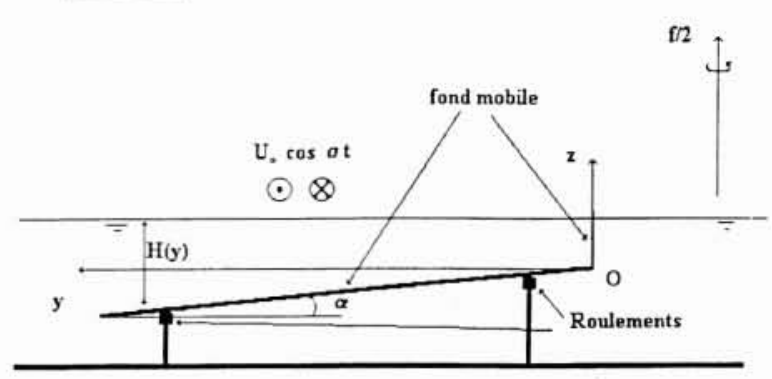

2. Dispositif expérimental.
$E_{\mathrm{k}}=v / f H^{2}=$ Frottement visqueux $/$ Coriolis

nombre d'Ekman

$R_{c}=U_{0} H / v=$ Inertie/Frottement visqueux

nombre de Reynolds

Nous ne respectons pas la similitude de Froude (effets d'ondes de gravité négligés). Bien que nous assurant que l'écoulement est suffisamment turbulent, la similitude de Reynolds n'est pas rigoureusement respectée.

Les mesures des vitesses sont réalisées à l'aide d'une batterie de sondes ultrasoniques fixées au fond mobile oscillant, disposées dans le plan $(x=0)$ et explorant régulièrement le champ des vitesse selon $(O y)$ et $(O z)$. Puis, nous analysons les signaux des composantes horizontales $u$ et $v$ en terme de décomposition harmonique :

$$
\begin{aligned}
u(y, z, t)=u_{0}(y, z) & +u_{1}(y, z) \cos \left(\sigma t-\phi_{1}(y, z)\right) \\
& +u_{2}(y, z) \cos \left(2 \sigma t-\phi_{2}(y, z)\right)+\cdots \\
v(y, z, t)=v_{0}(y, z) & +v_{1}(y, z) \cos \left(\sigma t-\psi_{1}(y, z)\right) \\
& +v_{2}(y, z) \cos \left(2 \sigma t-\psi_{2}(y, z)\right)+\cdots
\end{aligned}
$$

dont nous ne retenons que les deux premiers termes relatifs aux indices « 0 » (courants résiduels du second ordre) et « $1 »$ (courants oscillants du premier ordre). Il ne nous est pas possible de mesurer la vitesse verticale. Mais nous avons pu observer très clairement à l'aide de colorants le pompage d'Ekman à la côte s'effectuant dans une couche très mince dite de Stewartson (voir 2.2).

\subsection{Similitude. Aspect théorique}

Le choix d'une valeur significative de viscosité turbulente verticale sur le modèle, image de l'état de la turbulence, est difficile. Quelques expériences préliminaires nous ont conduit à choir une valeur de $v=0.03 \mathrm{~cm}^{2} / \mathrm{s}$. Nous fondant sur les approches de MAAS et al., nous choisissons une valeur de viscosité nature de l'ordre de $10^{-3} \mathrm{~m}^{2} / \mathrm{s}$, pour laquelle le modèle de distribution verticale de Prandle convient. Dans la suite, l'indice " $N$ " désignera les grandeurs «Nature", l'indice « $m$ " les grandeurs « modèle ". Nous noterons $[X]$ l'échelle associée à la variable $X:[X]=X_{m} / X_{N}$. Ainsi, $[v]=3 \cdot 10^{-3}$. Les valeurs nature des différents paramètres énumérés ciavant $\left(H_{N}=30 \mathrm{~m}, U_{0 N}=1 \mathrm{~m} / \mathrm{s}, \ldots\right)$ nous conduisent au choix suivant :

$$
\begin{aligned}
& {[H]=1.7 \cdot 10^{-3}} \\
& {[T]=1 /[\sigma]=1 / 1080} \\
& {[U]=0.17} \\
& {[\alpha]=14 .}
\end{aligned}
$$

Ces échelles déterminent les grandeurs dimensionnelles du modèle. Dans le cas d'un fond horizontal, nous obtenons : $H_{m}=5 \mathrm{~cm}, \Omega_{m}=0.042 \mathrm{rad} / \mathrm{s} \quad\left(T_{m}=150 \mathrm{~s}\right)$, $\sigma_{m}=0.10 \mathrm{rad} / \mathrm{s}\left(\tau_{m}=60 \mathrm{~s}\right)$, et $U_{0 m}=17 \mathrm{~cm} / \mathrm{s}$. Dans le cas d'un fond incliné, la pente $\alpha$ sur le modèle est telle que : $\tan \alpha=5 \%$. Dans ce cas, $H$ représentera la hauteur à la côte (en $y=0$ ), c'est-à-dire de l'ordre de $1 \mathrm{~cm}$. Nous avons également procédé à des expériences en faisant varier ces valeurs données par la similitude. Le tableau $n^{\circ} I$ résume les différentes expériences réalisées. 


\section{AELBRECHT, G. CHABERT D'HIÈRES}

Tableau 1. - Paramètres des différentes expériences.

\begin{tabular}{|c|c|c|c|c|c|}
\hline $\mathrm{N}^{*}$ & $T(\mathrm{~s})$ & $\tau(\mathrm{s})$ & $H(\mathrm{~cm})$ & $\tan \alpha$ & $U_{0}(\mathrm{~cm} / \mathrm{s})$ \\
\hline 1 & 300 & 200 & 5 & 0.00 & 3.9 \\
\hline 2 & 150 & 60 & 5 & 0.00 & 17.7 \\
\hline 3 & 150 & 60 & 1 & 0.05 & 17.1 \\
\hline 4 & 150 & 30 & 1 & 0.05 & 18.0 \\
\hline 5 & 75 & 60 & 1 & 0.05 & 16.0 \\
\hline
\end{tabular}

La formulation mathématique du problème nous permet de mieux comprendre quels termes dynamiques interviennent dans le processus étudié. Si l'on se place dans le repère lié au fond oscillant, on peut écrire (compte tenu que l'écoulement est indépendant de $x$ ) :

$$
\begin{gathered}
\frac{\partial(u+U(t))}{\partial t}+v \frac{\partial u}{\partial y}+w \frac{\partial u}{\partial z}-f v=v_{y} \frac{\partial^{2} u}{\partial y^{2}}+v_{z} \frac{\partial^{2} u}{\partial z^{2}} \\
\frac{\partial v}{\partial t}+v \frac{\partial v}{\partial y}+w \frac{\partial v}{\partial z}+f(u+U(t))= \\
=-\frac{1}{\rho} \frac{\partial p^{*}}{\partial y}+v_{y} \frac{\partial^{2} v}{\partial y^{2}}+v_{z} \frac{\partial^{2} v}{\partial z^{2}} \\
0=-\frac{1}{\rho} \frac{\partial p^{*}}{\partial z}-g \\
\frac{\partial v}{\partial y}+\frac{\partial w}{\partial z}=0 .
\end{gathered}
$$

Les conditions aux limites étant classiques: au fond $(z=0)$, condition de non glissement (vitesse nulle), ou de frottement linéarisé (vitesse de glissement) ; de même pour la limite latérale (en $y=0)$. En surface $(z=H)$, pas de contrainte (gradient de la vitesse nul). On écrit ce système d'équations en supposant l'hypothèse hydrostatique vérifiée $(w \ll u$ et $v$ ). Le changement de repère (lié au fond mobile) fait intervenir deux nouveaux termes $(\mathrm{d} U(t) / \mathrm{d} t$ et $f U(t))$ qui, une fois passés dans le second membre de (1.1) et (1.2), apparaissent comme deux termes de gradient de pression. Le terme $p^{*}$ inclut les autres termes de pression, y compris le terme de force centrifuge. PrandLE (1982) se place dans le cas infini (indépendance également selon $y$, et donc pas de vitesse verticale $w$ d'après l'équation de continuité), et ne tient pas compte des termes non-linéaires des équations (1.1) et (1.2). Il aboutit à une solution analytique de la structure verticale des courants oscillants (appelés $u_{1}$ dans le texte). TEE (1979 et 1980) calcule numériquement les termes résiduels et oscillants, reprenant la totalité des équations mais sans tenir compte des termes de diffusion horizontale dans la zone côtière (frottement visqueux), qui déterminent, dans notre cas, la structure de la couche limite latérale, et par conséquent le pompage d'Ekman. Ce pompage (mouvement vertical ascendant et descendant) s'opère le long de la paroi latérale dans une couche très mince appelée couche de Stewartson $\delta_{s}$ (voir PEDLOSKY,
1987) que l'on peut estimer par la relation : $\delta_{s}=E k_{h}^{1 / 2} / E k_{\mathrm{v}}^{1 / 4}\left(=E k^{1 / 4}\right.$ si les viscosités horizontale et verticale sont égales) clairement mise en évidence par injection de colorants. Lorsque l'écoulement laisse la côte à droite (fig. $3 a$ ), les particules fluides de la couche supérieure sont advectées vers la côte, descendent le long de celle-ci dans la couche d'épaisseur $\delta_{s}$, et transitent ensuite dans la couche d'Ekman. Ce mécanisme a pour effet de transporter une grande quantité de mouvement vers des régions (près de la côte) de faible quantité de mouvement, et par conséquent modifie (diminue) l'épaisseur de la couche limite latérale. Il faut noter dans ce cas la persistance de "l'overflow " classique d'une couche
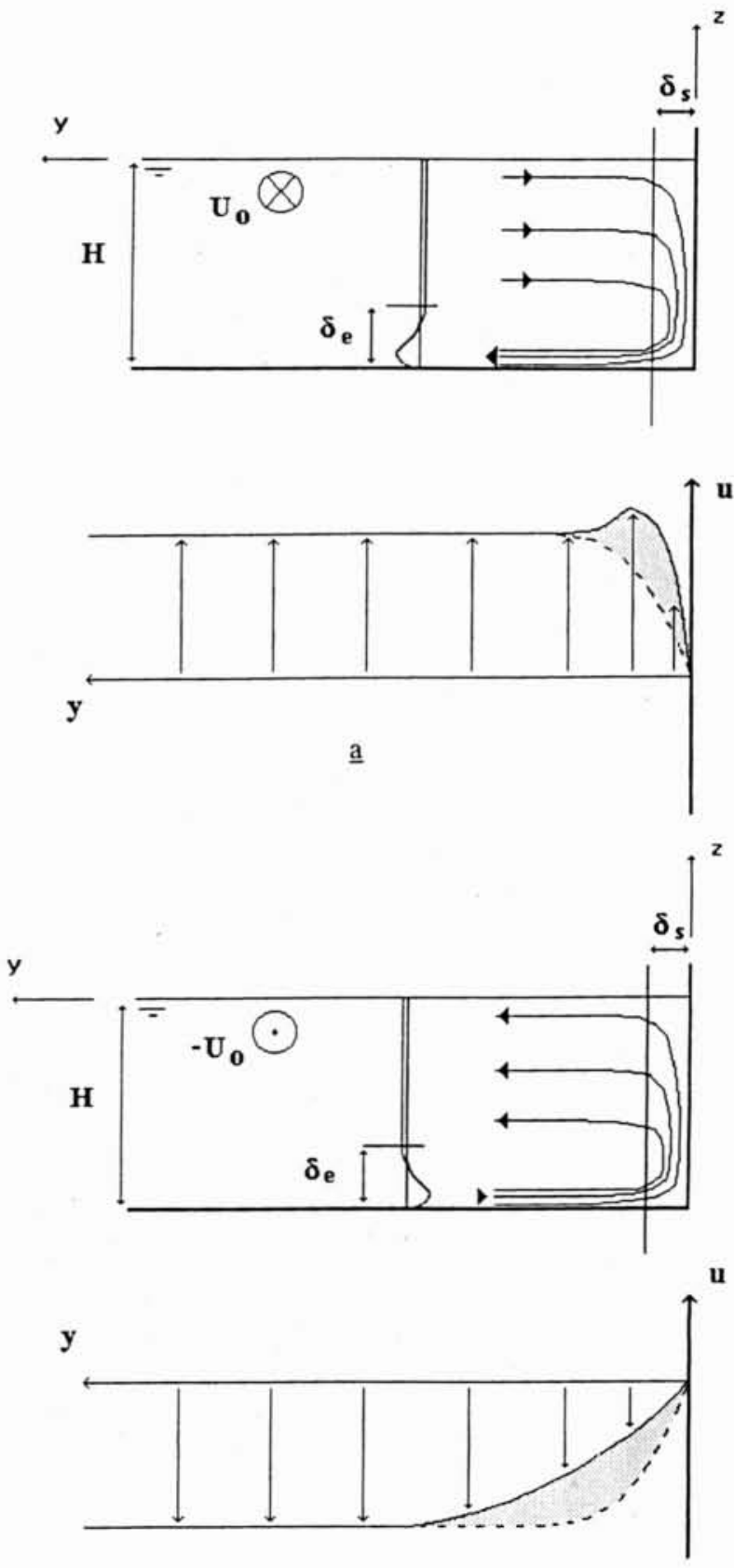

$\underline{b}$

3. Schéma explicatif du mécanisme de pompage d'Ekman. 
limite inertielle (dite « de Stokes »). Dans la configuration opposée (fig. $3 b$ ), ce mécanisme étire la couche limite latérale, et transporte des faibles quantités de mouvement plus au large. Sur un cycle de marée, la différence des épaisseurs de couches limites (parties grisées) entre la couche classique et la couche modifiée par pompage engendre un courant résiduel à la côte. Dans ce cas, on observe la disparition de l'overflow. Un modèle numérique aux équations primitives de ce processus a été entrepris en collaboration avec l'Université d'Arizona, dans le cas d'un écoulement laminaire (ZHANG et al., 1993).

\section{Résultats}

La représentation classique des vitesses par l'ellipse de courant donne une bonne idée de l'effet du processus : la figure $4 a$ montre clairement la dissymétrie de la couche limite latérale dans la couche supérieure suivant le sens de l'écoulement, conformément à l'explication donnée ci-dessus. Les figures $5 a$ et $5 b$ décrivent la structure verticale des ellipses du courant horizontal (rabattues sur la verticale) où l'on peut observer à la fois : l'inversion attendue du sens de rotation décrit par le vecteur vitesse à mi-profondeur; la décroissance de l'amplitude de la

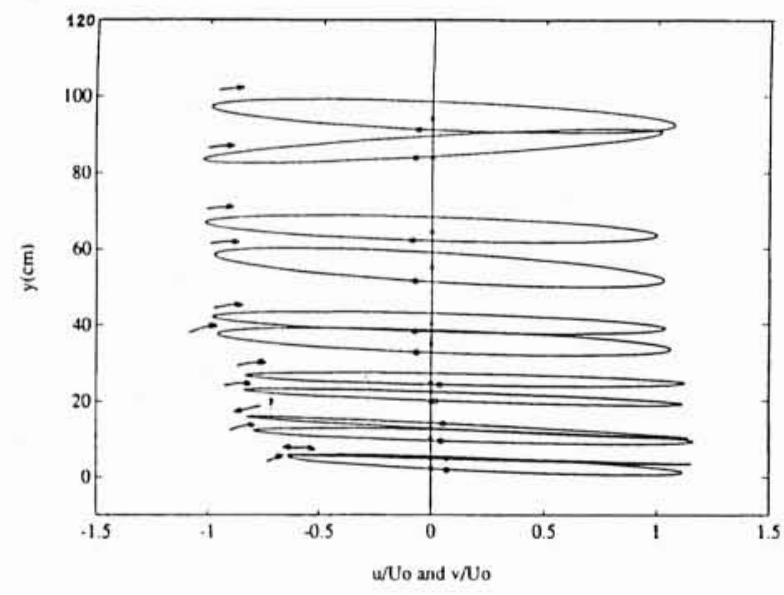

a

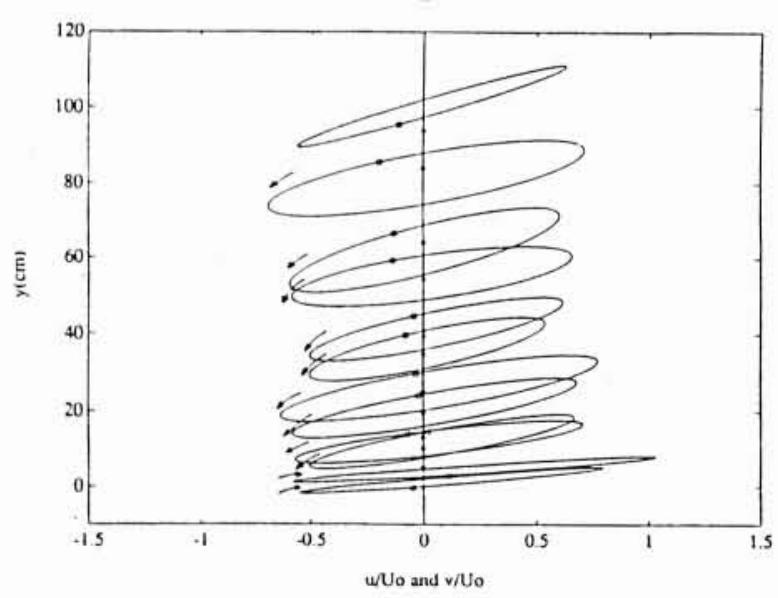

4. a: Structure horizontale des ellipses de courant en surface (exp. $\left.N^{\circ} 3\right) ; b$ : Structure horizontale des ellipses de courant au fond (exp. $N^{\circ} 3$ ).

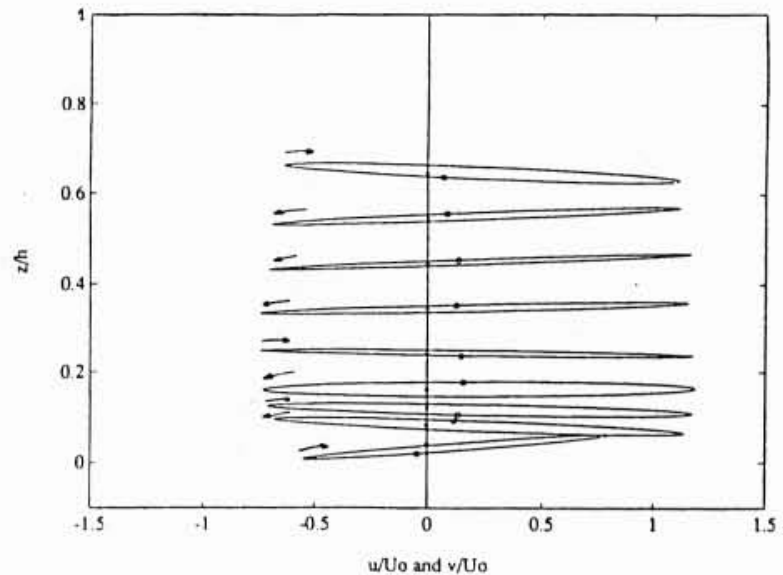

a

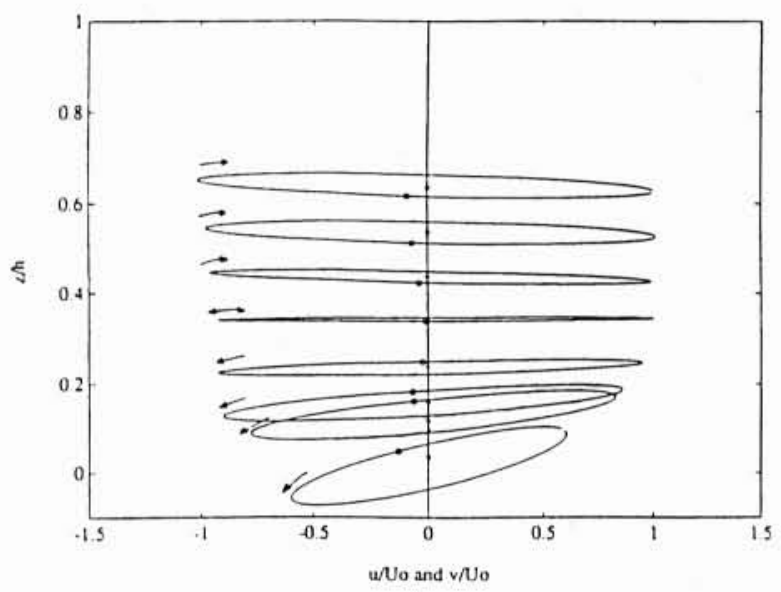

b

5. a : Structure verticale des ellipses de courant près de la paro latérale (exp. $N^{\prime 3}$ ) ; $b$ : Structure verticale des ellipses de courant loin de la paroi latérale (exp. $N^{*} 3$ ).

vitesse; et l'inclinaison de l'ellipse vers le fond. La structure tridimensionnelle du courant résiduel est décrite sur la figure 6 , sur laquelle nous avons représenté ce courant résiduel normalisé $u_{0} / U_{0}$ en fonction de la distance à la paroi $y$ et de la profondeur $z$ pour l'expérience $\mathrm{N}^{\circ} 3$ (cf. tabl. 1). Les échelles du laboratoire et leurs « équivalents nature » y sont indiqués. Le processus aboutit donc, dans des conditions de similitude appliquée à la nature, à la formation d'un courant résiduel d'une largeur d'environ $4 \mathrm{~km}$ et d'une amplitude maximale (près de la côte) de l'ordre de $20 \%$ de l'amplitude du courant de marée $\left(U_{0}\right)$. Le débit moyen côtier lié à ce processus est alors de l'ordre de $10000 \mathrm{~m}^{3} / \mathrm{s}$. D'un point de vue lagrangien, il faut ajouter à cette dérive eulérienne la dérive de Stokes, que l'on peut estimer, dans le cas d'une onde progressive, et pour un module de vitesse eulérienne $V$ constant, à $V^{2} /\left(2(g H)^{1 / 2}\right)$ dirigée dans les sens de propagation de l'onde (ZIMmerman, 1979). Pour nos conditions dans la nature, cette dérive de Stokes est de l'ordre de $3 \mathrm{~cm} / \mathrm{s}$ dirigée vers le Nord (laissant la côte à droite), ce qui représente de l'ordre de $3 \%$ de l'amplitude du courant de marée.

L'effet de la turbulence tend à diminuer ce phénomène, du fait d'une couche d'Ekman moins marquée (l'angle de déviation du vecteur vitesse devient plus faible). Néan- 


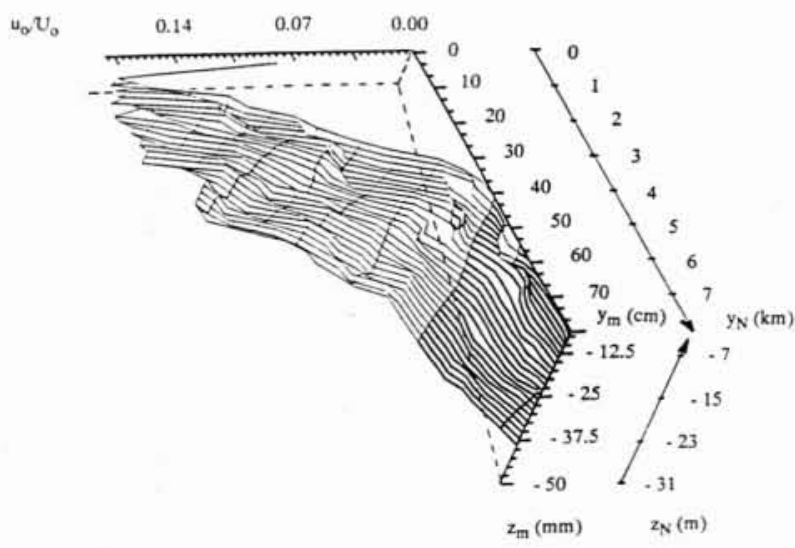

6. Profil 3-D du courant résiduel normalisé (expérience $N^{*} 3$ ).

moins, cet effet demeure et est à inclure dans toute étude de transport côtier, sans compter qu'il démontre une circulation tridimensionnelle que peu de modèles prennent généralement en compte. Revenons à l'application de nos résultats à la région de Boulogne $\mathrm{s} / \mathrm{mer}$. Le débit résiduel côtier que nous obtenons est certes plus faible que celui estimé pour tout le détroit du Pas-de-Calais. Mais il intervient dans une bande côtière où les concentrations de matière en suspension sont 4 à 5 fois celles des eaux centrales (voir $2^{\mathrm{c}}$ rapport annuel FLUXMANCHE, 1992). En conséquence, le débit moyen côtier de matières en suspension est important. Enfin, nous pouvons citer l'existence d'instabilités hydrodynamiques (dans les couches limites de fond et latérale) qui déterminent le mécanisme de transition vers un écoulement turbulent. Ces instabilités pourraient être à l'origine de fortes fluctuations de vitesse (notamment verticale) durant certaines phases de l'oscillation de marée, et provoquer des échanges verticaux (remise en suspension intense) dans la colonne d'eau. L'étude de ces instabilités est en cours actuellement.

\section{Conclusion}

Nous avons mis en évidence expérimentalement un processus de génération d'un courant résiduel côtier dans un écoulement supposé sinusoïdal au large. Ce processus de rectification, lié au pompage d'Ekman, peut se superposer à d'autres mécanismes de rectification (effet topographique) ou de génération de circulation résiduelle (effets du vent par exemple). Extrapolé à la nature, il engendre un flux résiduel d'eau côtière d'environ $10000 \mathrm{~m}^{3} / \mathrm{s}$ en moyenne, auquel il faut ajouter la dérive de Stokes si l'on s'intéresse au suivi des masses d'eau. Appliquée à la région de Boulogne s/Mer, ka prise en compte de ce transport moyen côtier est cruciale pour l'estimation du débit de matière en suspension qui transite par la bande côtière à travers le détroit du Pas-de-Calais. Enfin des instabilités hydrodynamiques dans les couches limites sont observées, dont l'effet probable sur les échanges d'énergie (verticaux notamment) est à l'étude actuellement.

Ce travail a été mené dans le cadre du projet européen FLUXMANCHE (MAST 0053-C), supporté par la Commission des Communautés Européennes.

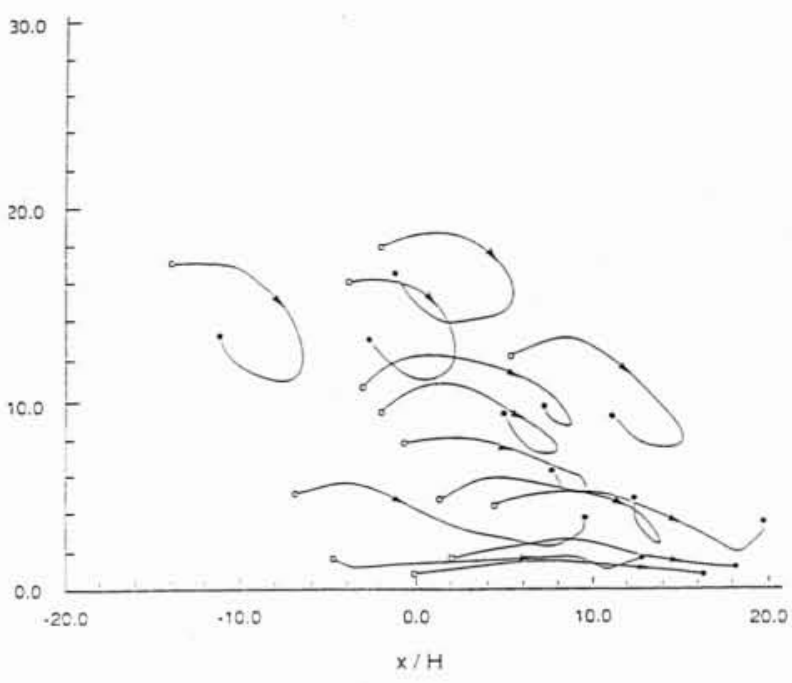

7. Tracé de trajectoires de flotteurs de surface (exp. $\left.N^{*} 1\right)$.

\section{Références}

Aelbrecht D., Chabert D'Hières $G$. et Zhang $X$. (1993). - Generation of a rectified current by interaction between coastal boundary layer and Ekman layer in a tidal motion. Oceanologica Acta, Actes du Symposium Manche, sous-presse.

FLUXMANCHE, prog. européen MAST 0053-C (1992). - Hydrodynamics and Biochemical Fluxes in the Eastern Channel: Fluxes into the North Sea. Second Annual Progress Report, June 1991-September 1992.

GARREAU P. \& MAZE R. (1992). - Tidal rectification and mass transport over a shelf break : a barotropic frictionless model. J. of Phys. Oceano., 22, 719-731.

MAAS L.R. \& VAN HAREN J.J. (1987). - Observations on the vertical structure of tidal and inertial currents in the central North Sea. J. of Marine Research, 42-2, 293-318.

Pedlosky J. (1987). - Geophysical Fluid Dynamics. 2nd edition, Springer Verlag.

PRANDLE D. (1982), - The vertical structure of tidal currents. Geophys. \& Astro. Fluid Dyn., 22, 29-49.

SAlomon J.C. \& BREton M. (1991). - Courants résiduels de marée dans la Manche. Oceanologica Acta. Actes du Colloque international sur I'environnement des mers épicontinentales, Lille, 20-22 mars 1990, vol. sp. 11.

TEE K.T. (1979), - The structure of three-dimensional tide generating currents. Part I: oscillating currents. J. of Phys. Ocea., 9, 930-944.

TEE K.T. (1980), - The structure of three-dimensional tide generating currents. Part II: residual currents. J. of Phys. Ocea., 10, 2035-2057.

Zhang X., Boyer D.L., Chabert D'Hiêres G. et Aelbrecht D. (1993). - Rectified flow of a Rotating fluid along a vertical side wall. Soumis à Physics of Fluids.

ZIMMERMAN J.T.F. (1978), - Topographic generation of residual circulation by oscillatory (tidal) currents. Geophys. \& Astrophys. Fluid Dyn., 11, 34-47.

Zimmerman J.T.F. (1979), - On the Euler-Lagrange transformation and the Stokes'drift in the presence of oscillatory and residual currents. Deep-Sea Research, 26A, 505520. 